\title{
Immunoglobulin heavy chain gene analysis in bone marrow biopsies and corresponding lymph node specimens: Dependency on pre-treatment, histological subtype and extension of B-cell lymphoma
}

\author{
MARGARETE ODENTHAL ${ }^{1 *}$, UDO SIEBOLTS ${ }^{1,2^{*}}$, KAREN ERNESTUS $^{1}$, \\ DANIEL DISSE $^{1}$, HANS PETER DIENES ${ }^{1}$ and CLAUDIA WICKENHAUSER ${ }^{1}$ \\ ${ }^{1}$ Institute of Pathology, ${ }^{2}$ Center for Molecular Medicine (CMMC), \\ University of Cologne, Kerpener Str. 62, D-50924 Cologne, Germany
}

Received October 17, 2007; Accepted December 14, 2007

\begin{abstract}
Bone marrow biopsies (BMB) are the conventional staging method for assessing marrow involvement by lymphoma. Morphological criteria provide basic data determining their dignity, but concerning microfocal infiltrates, these criteria are rather inaccurate. Here, by examination of immunoglobulin $\mathrm{H}(\mathrm{IgH})$ receptor rearrangement and comparison of medullar and nodular lymphoma sites, diagnostic reliability was improved. Employing non-nested $\mathrm{IgH}$ rearrangement analysis with FR3A and JHa consensus primers, B-cell clonality was assessed on glutardialdehyde fixed, decalcified BMB with and without lymphoma infiltration and on the corresponding lymph node specimens. Malignancy was confirmed when polymerase chain reaction (PCR) generated no more than two peaks and was observed in $60 \%$ of the medullar B-cell lymphoma. Comparison of lymph node tissues and BMB revealed an identical pattern in 50\% of the probes. In $25 \%$ of the cases a single clonal peak derived from the lymph node tissues was also observed in the BMB but was surrounded by additional peaks. Here, direct comparison of the data permitted determination of lymphoma in the BMB. Therefore, IgH FR3 PCR analysis is a suitable tool to examine small lymphoid infiltrates in $\mathrm{BMB}$, and direct comparison with corresponding nodal lymphoma can further facilitate estimation of their dignity.
\end{abstract}

Correspondence to: Dr Claudia Wickenhauser, Institute of Pathology, University of Cologne, Kerpener Str. 62, D-50924 Cologne, Germany

E-mail: c.wickenhauser@uni-koeln.de

\section{${ }^{*}$ Contributed equally}

Key words: lymphoma, immunoglobulin $\mathrm{H}$ receptor rearrangement, bone marrow biopsy

\section{Introduction}

There are still conflicting opinions over the distinction between benign (reactive) focal lymphoid aggregates and focal infiltrates of malignant lymphomas in BMB (1-4). Although in BMB particularly histotopography and cytomorphology of lymphoid infiltrates and an increase in reticulin fibers may lead to a certain suspicion concerning their dignity, the morphological evaluation lacks diagnostic reliability particularly concerning microfocal infiltrates and biopsies with inferior quality (5). In daily routine diagnostics, however, the hematopathologist is frequently confronted with this differential diagnosis especially with respect to lymphoma staging, as well as for assessing treatment response and/or restaging. In addition, in lymphoproliferative diseases without $\mathrm{LN}$ involvement, diagnosis can only be ascertained in suitable BMB.

IgH FR3 PCR analysis has already been used by others to check clonality of lymphoid infiltrates in BMB (6-12). However, until now the support of corresponding LN clonality analysis in diagnosis of BM involvement has not been investigated systematically. Therefore, in this retrospective study we evaluated the impact of this assay on glutardialdehyde (GA)-fixed, decalcified BMB especially with regard to small medullar lymphoid infiltrates. Data were then compared with those obtained from corresponding lymphoma-infiltrated LN tissues, and this assay facilitated the interpretation of BMB-derived data. When the histomorphology of the BMB was compared with the clonality data, peritrabecular position and increase of reticular fibers were the most significant features indicating malignancy. Prerequisites for a high quality hematological diagnosis therefore incorporate the optimal histological processing of $\mathrm{BMB}$, IgH rearrangement analysis and, ideally, comparison of nodal and medullar lymphoma sites.

\section{Materials and methods}

Tissue samples. Paraffin wax-embedded specimens of BMB and LN tissue were analyzed from a total of 40 patients 
whose files were archived at the Institute of Pathology of the University of Cologne, Germany.

Selection of BMB occurred in a three-step procedure. Firstly, patients with nodal ascertained chronic lymphatic leukemia of B-cell type (B-CLL), mantle cell lymphoma (MCL) and follicular lymphoma (FL) grade 1 to grade 2 were selected employing the WHO classification system. From these patients, BMB drawn from our files were stained, and specimens with lymphoid infiltrates were subdivided according to the proportion of the infiltrates with respect to the hematopoietic area. Groups with infiltrate densities of 5 , $10,15,30$, and $80 \%$ were chosen. Each group consisted of five patients. LN specimens of the selected patients were analyzed separately. Histological appraisal including immunohistochemistry was performed independently by two experienced hematopathologists.

In addition, we chose each of five BMB infiltrated by malignant plasma cell myeloma (MM) and immunocytoma (IC). In these cases, monotypic light chain expression was ascertained by immunohistochemistry. The biopsies were subdivided according to their proportion of tumor cell population. Finally, five BMB fixed in buffered $4 \%$ formalin fixative were analyzed.

Negative controls comprised five LN specimens with follicular hyperplasia as well as ten BMB from patients with lymphoid infiltrates and well-known immunomodulatory/ infectious diseases like autoimmune thyroiditis and hepatitis $\mathrm{C}$ or unclear anemia. As a further positive control we chose the L1236 Hodgkin's lymphoma cell line (13).

Processing of $B M B$ and histological staining. The main group of BMB was fixed in a solution of $30 \mathrm{ml}$ formalin (at least 35\%, Merck, Darmstadt, Germany), $20 \mathrm{ml}$ glutardialdehyde 25\% (Merck) and $15.8 \mathrm{~g}$ calcium acetate (Merck) and $1000 \mathrm{ml}$ Aqua dest (Schaefer fixative). Decalcification was conducted for 3-4 days in 10\% Tris-buffered ethylenediaminetetraacetic acid (EDTA; Merck) pH 7.2-7.4. The second group of BMB was fixed in buffered $4 \%$ formalin fixative while decalcification was also conducted in EDTA. Sections were stained with Giemsa, naphthol-AS-D-chloroacetate esterase and Gomori's stain for reticulin fibers. Five BMB (2 patients with MCL, 2 with B-CLL and 1 patient with FL grade I) were fixed in buffered $4 \%$ formalin fixative.

Processing of LN specimens and histological staining. All lymph node specimens were fixed in buffered $4 \%$ formalin fixative and paraffin embedded. Slides were stained with H\&E and Giemsa.

Immunohistochemistry. Deparaffinized 3 to $5 \mathrm{~mm}$ sections were rehydrated, and heat-induced epitope retrieval was performed by microwave method in $0.1 \mathrm{M}$ sodium citrate buffer (Merck), pH 6.0. Monoclonal antibody collection included L26 against CD20 (Dako, Hamburg, Germany; dilution 1:100) as a pan-B-cell marker, F7.2.38 against CD3 (Dako; 1:100) as pan-T-cell marker, R10-21-F3 against light chain $\kappa$ and N10/2 against light chain $\lambda$ (both from Dako). The specimens were immunostained according to the ABC method. The secondary biotinylated antibody and the strepatavidin/alkaline phosphatase conjugate were applied according to the manufacturer's instructions (BioGenex, San Ramon, CA, USA). New fuchsin (Serva, Heidelberg, Germany) and naphthol-AS-BI phosphate (Sigma) were used as chromogens. Appropriate positive and negative control stainings were run simultaneously.

Extraction of DNA from tissue sections. Extraction of DNA was performed as described previously (14). In brief, one to three $8 \mu \mathrm{m}$ paraffin wax-embedded sections were dewaxed in xylene by incubation at $65^{\circ} \mathrm{C}$ for $5 \mathrm{~min}$. After vortexing, samples were centrifuged at 13,000 rpm. The procedure was repeated three times. The dewaxed sections were then washed in $500 \mu 1100 \%$ ethanol. After lysis in $200 \mu 1$ proteinase $\mathrm{K}$ buffer $[500 \mu \mathrm{g} / \mathrm{ml}$ proteinase $\mathrm{K}$ (Gibco, Gaithersburg, MD, USA), $50 \mathrm{mM}$ Tris/ $\mathrm{HCl}, \mathrm{pH} 7.4$, and $5 \mathrm{mM}$ EDTA, pH 8.0], nucleic acids were extracted by phenol/ chloroform and subsequently precipitated with $300 \mathrm{mM}$ sodium acetate and isopropanol. Each series of DNA extractions included sections taken from blocks of pure paraffin wax, which were cut between. The isolated DNA was dried, re-suspended in $25 \mu 1 \mathrm{H}_{2} \mathrm{O}$ and stored at $-20^{\circ} \mathrm{C}$.

Primers for $\beta$-globin PCR analysis. The quality of the DNA was evaluated by amplifying fragments of the human $\beta$-globin locus (forward, ACA-CAA-CTG-TGT-TCA-CTA-GC and reverse, CAA-CTT-CAT-CCA-CGT-TCA-CC; length of the amplificate, $109 \mathrm{bp}$ ).

Primers for IgH PCR. PCR analysis of the IgH gene involved the use of a consensus primer pair with the upstream primer being homologous to a $\mathrm{V}$ segment and the downstream primer annealing to a $\mathbf{J}$ segment. In detail, performing a non-nested PCR, a consensus FR3A primer was used as upstream primer (15), and a consensus JHa primer as downstream primer $(16,17)$ : FR3A primer (5' IRD 800 fluorochrome endlabeled), 5'-ACA CGG C(CT) (GC) TGT ATT ACT GT-3'; JHa primer, 5'-ACC TGA GGA GAC GGT GAC C-3'.

PCR approach and analysis. A hot start PCR approach was chosen. Analysis was performed in a thermocycler (Biometra). The reaction mix $(50 \mu \mathrm{l})$ contained $1.5 \mu 1,10 \mu \mathrm{M}$ of each primer, $10 \mu 1,1 \mathrm{mM}$ each of dNTPs, $5 \mu 110 \mathrm{X}$ concentrated PCR buffer $\left(25 \mathrm{mM} \mathrm{MgCl}_{2}\right.$ in $100 \mathrm{mM}$ Tris$\mathrm{HCL}$ and $\left.500 \mathrm{mM} \mathrm{KCl} \mathrm{pH} 8.3,20^{\circ} \mathrm{C}\right) 0.2 \mu 1$ of Taq DNA polymerase (5 units/ $\mu \mathrm{l}$ ) and $1.5 \mu \mathrm{l}$ genomic DNA and $50 \mu \mathrm{l}$ $\mathrm{H}_{2} \mathrm{O}$ (Merck). A PCR cycle consisted of annealing for $1 \mathrm{~min}$ at $52^{\circ} \mathrm{C}$, extension for $1 \mathrm{~min}$ at $72^{\circ} \mathrm{C}$, and denaturation for $1 \mathrm{~min}$ at $94^{\circ} \mathrm{C}$. Preceding each round, the PCR reaction was heated to $94^{\circ} \mathrm{C}$ for $5 \mathrm{~min}$, and after each round a final extension step of $5 \mathrm{~min}$ at $72^{\circ} \mathrm{C}$ was performed (40 cycles). Each experiment was replicated and contained a tube without DNA template (as a negative control), a tube whose template was DNA from a patient with ascertained MM, and native material from the L1236 cell line (positive controls).

Agarose gels. Electrophoresis was performed with PCRamplified material $(10 \mu \mathrm{l})$ in 3\% agarose gels in TAE buffer at 125 volts for $40 \mathrm{~min}$, and the DNA was visualized under short wavelength ultraviolet light after ethidium bromide staining of the gel. 

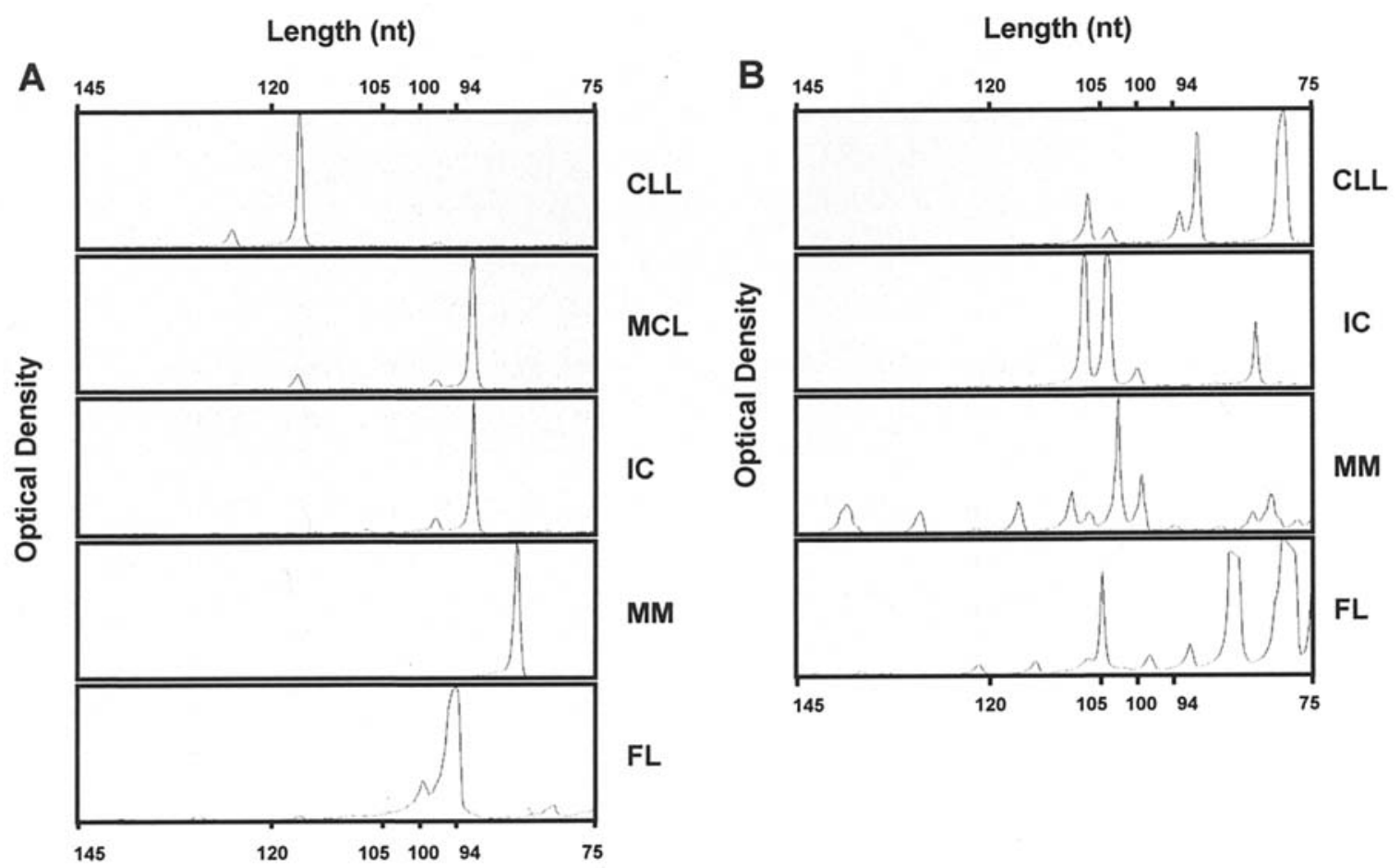

Figure 1. Computer-assisted fragment length analysis of IgH FR3 PCR amplification products from bone marrow biopsies (BMB). (A) A clear peak at defined nucleotide length indicating monoclonality of lymphoid infiltrates is visible for the different lymphoma entities. CLL, chronic lymphatic leukemia; MCL, mantle cell lymphoma; and IC, immunocytoma; MM, multiple myeloma; and FL, follicular lymphoma. (B) A multi-peak pattern indicates several B-cell subclones. The abscissa indicates the length of the amplicons in nucleotides (nt); the ordinate displays relative optical density.

Polyacrylamide gels. PCR products were run on $6 \%$ nondenaturating polyacrylamide gel electrophoresis (PAGE) overnight at $50^{\circ} \mathrm{C}$ and $1500 \mathrm{~V} / 41 \mathrm{~cm}$ employing an automated sequencer (LI-COR DNA-Analyzer Gene Reader 4200, MWG-Biotech, Ebersberg). The resolution of the PAGE was sufficient for detection of one base differences of amplicons, and genescan analysis of all samples was performed for assessment of IgH-specific PCR products. Illustration of the PAGE results as electropherograms was realized by measurement of optical densities within the gels employing the Scion Image Software release alpha 4.0.3.2 software (Scion Corp., Frederick, MD, USA).

Sequencing of the PCR products. PCR results with consensus primers were validated using sequence-specific primers labeled with the IRD 800 fluorescence dye (MWGBiotech). DNA bands of amplicons were cut from the gel and extracted using the Qiaex Kit (Qiagen, Hilden, Germany). The $100 \mu \mathrm{l}$ eluates were precipitated with $300 \mu \mathrm{M}$ sodium acetate, glycogen, and two volumes of ethanol, and the DNA was redissolved in $10 \mu 1 \mathrm{H}_{2} \mathrm{O}$. Two microliters of each sample was taken for PCR sequencing using the IRD 800-labeled JHa-seq primer: 5'-IRD 800-ACC TGA GGA GAC GGT GAC C-3'. Sequence analysis was carried out on a LI-COR DNA analyzer Gene Reader 4200. The sequence products were compared with the published data of the NCBI database by BLAST analyses to exclude amplification of a false amplicon.

\section{Results}

IgH rearrangement analysis in GA-fixed decalcified $B M B$. Prior to clonality analysis, PCR analysis for human B-globin was performed as a control for DNA extraction and delivered definite bands in all samples under study. Performing nonnested $\mathrm{IgH}$ rearrangement analysis with FR3A and JHa consensus primers, analysis of all ten $\mathrm{BMB}$ with reactive lymphoid infiltrates and 15 out of $25(60 \%)$ GA-fixed BMB with either medullar ascertained B-cell lymphoma or nodular ascertained B-cell lymphoma and suspicious lymphoid infiltrates in the corresponding $\mathrm{BMB}$, resulted in a distinct band in the agarose gels, respectively. In the latter group 18 out of 25 cases $(72 \%)$ presented definite amplicon visible by polyacrylamide gel electrophoresis. Of these cases, in 14 out of $18 \mathrm{BMB}$, employing electropherogram analysis, $\operatorname{IgH}$ rearrangement analysis resulted in no more than two main peaks (Fig. 1A). Specifically, in two cases out of five FL, in five cases out of five MCL, in two cases out of five B-CLL, in two cases out of five MM and three cases out of five IC a clonal rearrangement with no more than two bands was detected. The other four cases, two cases of FL, one case of B-CLL and one of MM presented three and more irregularly arranged peaks according to electropherogram analysis (Fig. 1B). In 7 out of 25 BMB with ascertained infiltration by B-cell lymphoma no peaks were visualized (Table I). In all cases with reactive lymphoid infiltrates a typical Gaussian distribution of the peak pattern was observed.

In individual cases, distinct amplicons visible in the agarose gels were cut out, extracted, and sequenced. The sequences achieved were compared with the published data of the NCBI database by BLAST analyses, and known VDJ regions were detected in all cases.

Outcome of $\mathrm{IgH}$ rearrangement analysis is independent of lymphoma extension in BMB. When reliability of the method 
Table I. Correlation between proportion of medullar B-cell infiltrates and clonality.

\begin{tabular}{lccccc}
\hline & $5 \%$ & $10 \%$ & $15 \%$ & $30 \%$ & $>80 \%$ \\
\hline FL & $1 / 1$ & $0 / 1$ & $0 / 1$ & $1 / 1$ & $0 / 1$ \\
MCL & $1 / 1$ & $1 / 1$ & $1 / 1$ & $1 / 1$ & $1 / 1$ \\
B-CLL & $1 / 1$ & $0 / 1$ & $0 / 1$ & $1 / 1$ & $0 / 1$ \\
MM & $0 / 0$ & $1 / 1$ & $0 / 1$ & $0 / 2$ & $1 / 1$ \\
IC & $0 / 0$ & $1 / 1$ & $1 / 1$ & $0 / 1$ & $1 / 2$ \\
\hline
\end{tabular}

Medullar infiltration of nodal ascertained low-grade malignant B-cell lymphoma and each of five cases of malignant plasma cell myeloma (MM) and of LP-immunocytoma (IC). The outcome of clonality analysis depends on histological subtype of lymphoma but not on the density of lymphoma infiltrates within the bone marrow. FL, follicular lymphoma; B-CLL, chronic lymphatic leukemia of B-cell type; MCL, mantle cell lymphoma. The degree of infiltrate per hematopoietic area is labeled horizontally. Cases with one or two peaks/overall cases are described for the various subgroups.

was evaluated, dependency of lymphoma extension in BMB on the significance of $\mathrm{IgH}$ rearrangement analysis was tested. Lymphoma infiltrates ranging from 5 to $80 \%$ lymphoid cells per hematopoietic area were compared. As described in Fig. 2 $\mathrm{IgH}$ rearrangement analysis of medullar mantle cell lymphoma infiltrates always indicated malignancy. Concerning BM infiltration by ascertained germinal center or post-germinal center lymphoma, malignancy was proved in a subset of cases, irrespective of the lymphoma extension (Table I). In all assays, clonality was detectable even in medullar infiltrates representing less than $5 \%$ of the hematopoietic area.

Peritrabecular position and aggregation of reticular fibers are the most important histological parameters indicating microfocal B-cell lymphoma infiltration in BMB. When data of IgH rearrangement analysis were obtained, histology and specific peculiarities of lymphoid infiltrates within the BMB were reevaluated. In MM biopsies and in most biopsies infiltrated by IC, immunohistochemistry of light and heavy chain expression turned out to be a very valid marker indicating malignancy (Fig. 3A and B). However, immunohistochemical analysis of $\mathrm{B}$ - and T-cell distribution failed to be a clear-cut parameter as did evaluation of cytological details in B-CLL, FL and MCL (Fig. 3C and D). Concerning small infiltrates $(<15 \%)$ the most significant histological parameters were peritrabecular position and aggregation of reticular fibers (Fig. 3E and F).

Comparison of IgH rearrangement analysis in nodal and medullar lymphoma infiltrates facilitates interpretation of clonality curves. Of all patients suffering from B-CLL, MCL and FL, nodal involvement was histologically ascertained. In this approach $\operatorname{IgH}$ rearrangement of each of the five cases was analyzed in the LN tissue as well as in the affected BMB. Clonality curves were compared and initially most conspicuous difference resulted in the clear peaks derived from LN tissue and more shaky peaks of minor quality in

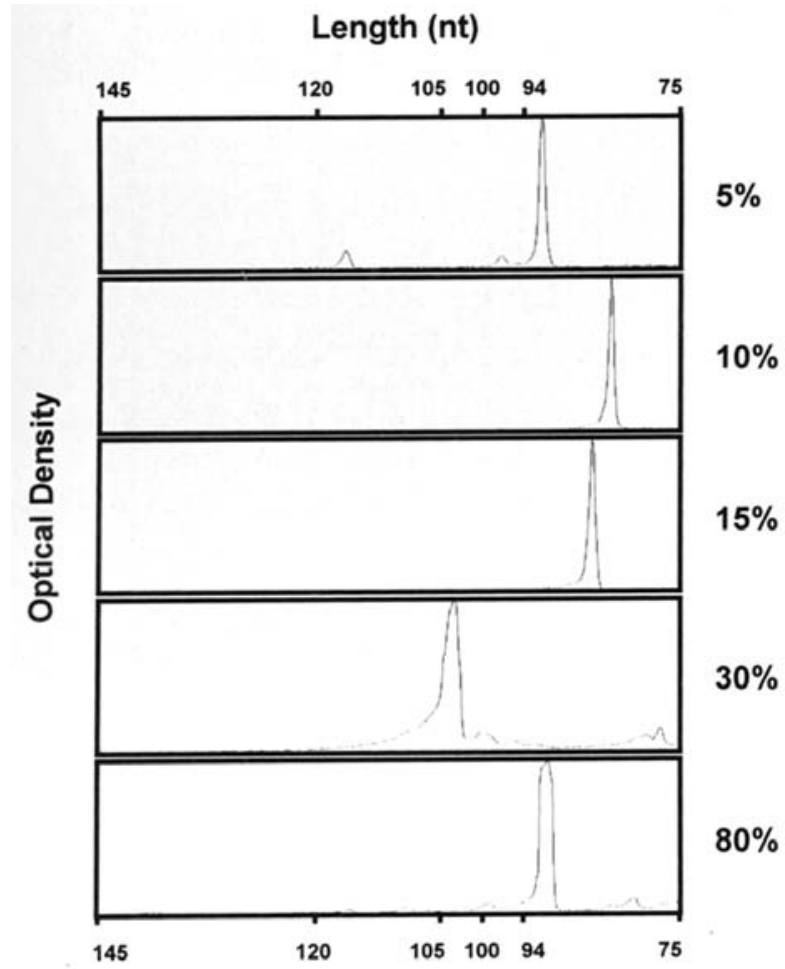

Figure 2. Computer-assisted fragment length analysis of IgH FR3 PCR amplification products from bone marrow biopsies (BMB). Five cases of nodular ascertained mantle cell lymphoma (MCL) with bone marrow infiltration were analyzed. Clonality of small infiltrates $(5 \%)$ was also detected. The extent of lymphoma infiltrates is indicated on the right side of the figure. The abscissa indicates the length of the amplicons in nucleotides $(\mathrm{nt})$; the ordinate displays relative optical density.

most of the GA-fixed BMB (Fig. 4A and C). In all cases where IgH PCR did not produce definite amplicons, this result was observed congruently in $\mathrm{BMB}$ and the corresponding LN tissues (4 out of 15 cases). A congeneric pattern with no more than two peaks was observed in 4 out of 15 cases (Fig. 4A). In 4 out of 15 cases, peaks appeared at the same position in both sources, but prominence of these peaks was different and additional peaks appeared in the BMB-derived lymphoma tissues (Fig. 4B). In 3 out of 15 cases the curve progression was largely different when both tissues were compared, although a slightly congeneric accordance still occurred (Fig. 4C; Table II).

Concerning non-neoplastic lymphoid tissue, a typical Gaussian distribution was observed in all of the $5 \mathrm{LN}$ tissues under study and in all 10 BMB with non-neoplastic lymphoid infiltrates (Fig. 4D).

Replacement of GA fixation by buffered formalin improves quality of IgH rearrangement analysis. To evaluate whether the shaky peaks following PAGE analysis of $\mathrm{IgH}$ rearrangement analysis were induced by GA fixation or decalcification, GA fixation was replaced by fixation in buffered formalin in a small collection of BMB. With the application of this procedure, the quality of the peak pattern increased. Following this procedure, as demonstrated in Fig. 5, curve progression in $\mathrm{BMB}$ and the corresponding $\mathrm{LN}$ tissues was of identical quality in these cases. 

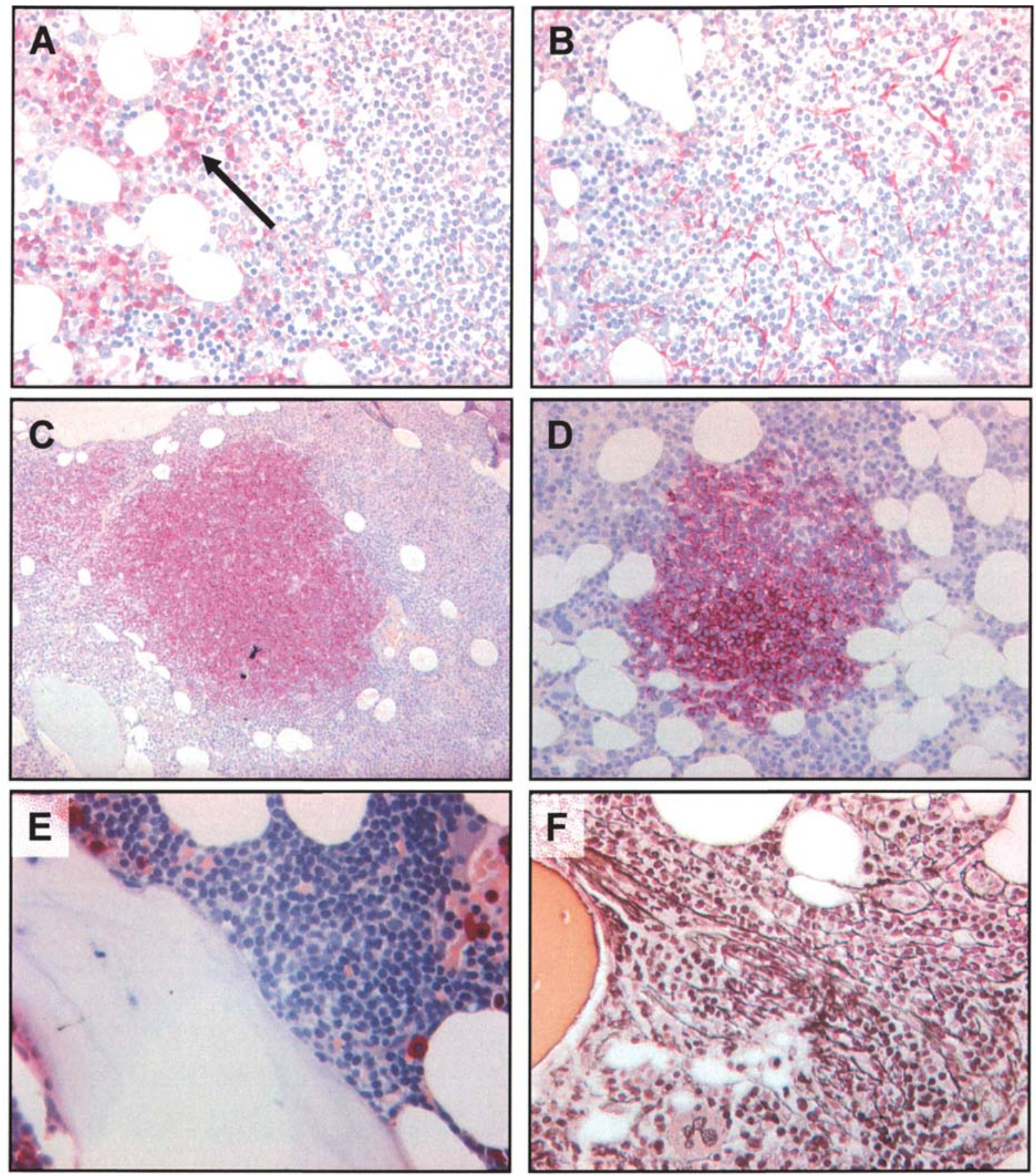

Figure 3. Histochemical analysis of lymphoid infiltrates in bone marrow biopsies (BMB). Detection of $\kappa$ (A) and $\lambda$ (B) light chain expression clearly indicates monoclonal plasma cell population within the infiltrate (arrow). Lymphonodular infiltrate of chronic lymphatic leukemia (B-CLL) with a mixed pattern of (C) CD20-positive B-cells and (D) CD3-positive T-cells. Here, immunohistochemistry does not contribute to the determination of dignity. (E) Chloracetatesterase staining of BMB reveals a small lymphoid infiltrate in peritrabecular position indicating malignancy in a patient with nodular ascertained mantle cell lymphoma (MCL). (F) Gomori silver staining of the same case. The increase in reticular fiber density also discloses malignancy.

Table II. Comparison of the clonality pattern between nodal and medullar lymphoma.

\begin{tabular}{lcccc}
\hline & Congeneric pattern & Identical peaks, different background & Different pattern & No amplificon \\
\hline FL & $0 / 5$ & $1 / 5$ & $2 / 5$ & $2 / 5$ \\
MCL & $4 / 5$ & $1 / 5$ & $0 / 5$ & $0 / 5$ \\
B-CLL & $0 / 5$ & $2 / 5$ & $1 / 5$ & $2 / 5$ \\
\hline
\end{tabular}

Comparison between IgH rearrangement analysis of the nodal and medullar lymphoma sites of individual patients. FL, follicular lymphoma; B-CLL, chronic lymphatic leukemia of B-cell type; and MCL, mantle cell lymphoma. Cases per overall cases are described for the various subgroups. 

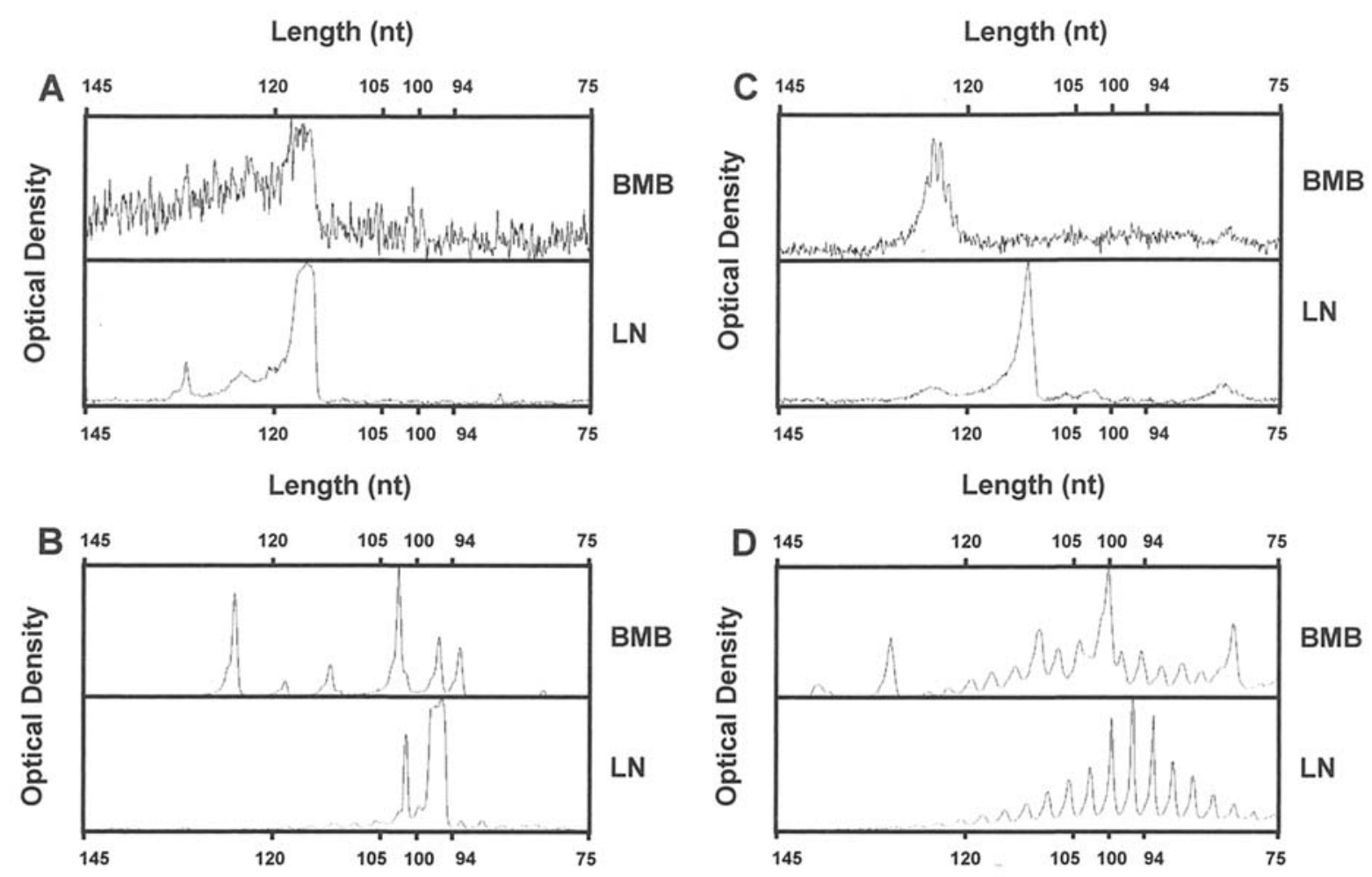

Figure 4. Computer-assisted fragment length analysis of IgH FR3 PCR amplification products. Analysis of corresponding bone marrow (BMB) and lymph node (LN) infiltrates. (A) Congruent pattern in a case of B-CLL. Shaky pattern in the BM analysis indicates inferior DNA quality following glutardialdehyde fixation. (B) Multi-peak pattern in the BMB-derived lymphoid tissue while analysis of the LN tissue revealed two clear peaks indicating clonality (case with FL). In this case, comparison of both curves facilitates interpretation of the data. (C) In other samples (here case with B-CLL) peaks at different nucleotide length indicate dominance of diverse clones at different tumor sites, although a slight elevation at the position of the main peak at the BMB is also seen in the LN analysis. (D) Gaussian distribution of amplification products in normal LN tissue (below) and also a multi-peak pattern in the BMB with reactive lymphoid infiltrates. The abscissa indicates the amplicon length in nucleotides (nt); the ordinate displays relative optical density.

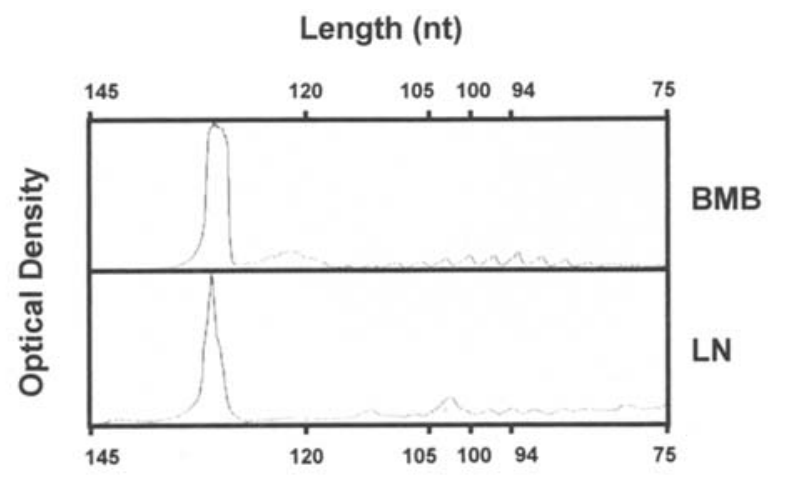

Figure 5. Computer-assisted fragment length analysis of IgH FR3 PCR amplification products. When glutardialdehyde fixative was replaced by buffered formalin, comparison of electropherograms from lymph node (LN) tissues and bone marrow biopsies (BMB) revealed identical quality of the curves in a case with chronic lymphatic leukemia (B-CLL). The abscissa indicates the amplicon length in nucleotides (nt); the ordinate displays relative optical density.

\section{Discussion}

Morphologic examination is the generally accepted method for determining bone marrow involvement by lymphoma in patients evaluated for extent of disease at diagnosis and after therapy. A clear-cut distinction between benign lymphoid infiltrates and focal infiltration by low-grade B-non-Hodgkin's lymphoma is possible in many cases when histotopography of lymphoid aggregates together with cytomorphology and density of reticular fibers are evaluated (5). Unfortunately, immunophenotyping in these cases is only of limited use because GA-containing fixatives allow the usage only of a limited panel of antibodies and because immunohistochemical determination of light or heavy chain restriction is only possible in a subset of lymphoma entities (5). A diagnostic grey zone therefore affects primarily very small lymphoid infiltrates and $\mathrm{BMB}$ of inferior quality.

Molecular methods for determining clonality provide alternative techniques for evaluating suspicious or indeterminate lymphoid infiltrates and have the advantage of increased specificity and decreased subjectivity. The consensus PCR protocols can be effectively used in routine molecular diagnostic laboratories and yield highly reproducible results with no reported false-positive results. However, their major drawback is an increased false-negative rate. Possible technical reasons for false-negative results have been reviewed $(18,19)$. In certain clonal populations, despite optimal reaction conditions, the consensus primers may lack sufficient homology to anneal with the pertinent $\mathrm{IgH}$ regions because of somatic hypermutation or ongoing mutations. Other reasons include competitive amplification of DNA from rearranged reactive bystanders, interfering chromosomal translocations or deletions. Although increasing the number of amplification cycles may enhance detection in some specimens, excessive cycling can result in increased polyclonal back- 
ground smearing and nonspecific bands that complicate assay interpretation, because the polyclonal smear may mask a monoclonal band or a nonspecific band may mimic a clonal band (18). Quantitative mRNA analysis of light chain expression via real-time PCR assay is well suitable for the analyses of highly mutated germinal centre/post-germinal centre lymphoma (20). However, the diagnostic impact is restricted due to poorly preserved RNA and susceptibility to the extent of clonal medullar expansion and reactive background. Furthermore, only the discrimination of distinct rearrangement length distribution enables the investigator to directly compare medullar and nodular clonality profiles.

Performing non-nested $\operatorname{IgH}$ rearrangement analysis with FR3A and JHa consensus primers in GA-fixed BMB, a clonal rearrangement could be detected in $60 \%$ of the probes with lymphoma involvement, dependent on the lymphoma subtype. This finding is within the normal range as described by others when analyzing neoplastic LN tissues. When BMB were reevaluated with regard to the results of the clonality data, peritrabecular position of lymphoid infiltrates and increases in fiber density were the most significant parameters indicating clonal processes. In this context, as described before, the adoption of the naphthol-AS-D-chloroacetate esterase is a prerequisite for the detection of rather small infiltrates (5). Hence, the careful morphological examination of BMB will continue to be valuable in the future, although the diagnostic certitude decreased when lymphoma extension was low. Therefore, evidence of monoclonality in BMB with a lymphoma extension of $<5 \%$ clearly indicates that $\operatorname{IgH}$ rearrangement analysis must be implemented in high quality hematological diagnostics. In this context it is important to state that patients with autoimmune diseases might also reveal clonal B-cell proliferation in the bone marrow due to autoantigen stimulation (10). Therefore BMB-derived clonality data from patients with autoimmune diseases have to be interpreted with utmost care and in context with clinical and histomorphological data.

When lymphoma infiltrates of LN tissues and the corresponding $\mathrm{BMB}$ were compared, the inferior quality of DNA derived from GA-fixed BMB was apparent. In contrast, concurrent molecular testing of bone marrow lymphoid infiltrates derived from biopsies fixed in buffered formalin identified DNA with identical quality as observed in the corresponding nodal lymphoma tissues.

Our data further indicate that a direct comparison of clonality data from LN tissues and BMB can facilitate the interpretation of the analysis, irrespective of the processing of the BMB. Particularly the appraisal of additional peaks derived from polyclonal B-lymphoid background in BMB is much clearer when employing this comparative method.

Using bone marrow aspirates, false-negative results in clonality analysis are rather frequent due to the failure to aspirate sufficient lymphoma cells (21-23). Therefore the method presented here is clearly superior to the strategy of others examining the BMB solely for morphological analysis and smears for further molecular clonality analysis $(18,24,25)$.

With an overall sensitivity of non-nested $\operatorname{IgH}$ rearrangement analysis of $60 \%$ in the BMB the values are slightly worse than described for the analysis of nodal lymphoma, probably due to the inferior DNA quality of this GA fixative and also the decalcified material (26). Adaptation of the BIOMED-2 approach at this point has the potential to further increase sensitivity (27).

With an excellent specificity, our data clearly show that IgH FR3 PCR analysis of BMB is a reliable tool to analyze small lymphoid infiltrates of uncertain dignity which can be further improved by comparison with the corresponding nodal lymphoma. A systematic adoption of this approach must be endorsed in high quality hematological diagnostics.

\section{References}

1. McKenna RW and Hernandez JA: Bone marrow in malignant lymphoma. Hematol Oncol Clin North Am 2: 617-635, 1988.

2. Salisbury JR, Deverell MH and Cookson MJ: Threedimensional reconstruction of benign lymphoid aggregates in bone marrow trephines. J Pathol 178: 447-450, 1996.

3. Faulkner-Jones BE, Howie AJ, Boughton BJ and Franklin IM: Lymphoid aggregates in bone marrow: study of eventual outcome. J Clin Pathol 41: 768-775, 1988.

4. Schmid C and Isaacson PG: Bone marrow trephine biopsy in lymphoproliferative disease. J Clin Pathol 45: 745-750, 1992.

5. Thiele J, Zirbes TK, Kvasnicka HM and Fischer R: Focal lymphoid aggregates (nodules) in bone marrow biopsies: differentiation between benign hyperplasia and malignant lymphoma - a practical guideline. J Clin Pathol 52: 294-300, 1999.

6. Brinckmann R, Kaufmann O, Reinartz B and Dietel M: Specificity of PCR-based clonality analysis of immunoglobulin heavy chain gene rearrangements for the detection of bone marrow involvement by low-grade B-cell lymphomas. J Pathol 190: 55-60, 2000.

7. Lassmann S, Gerlach UV, Technau-Ihling K, Werner M and Fisch P: Application of BIOMED-2 primers in fixed and decalcified bone marrow biopsies: analysis of immunoglobulin $\mathrm{H}$ receptor rearrangements in B-cell non-Hodgkin's lymphomas. J Mol Diagn 7: 582-591, 2005.

8. Pittaluga S, Tierens A, Dodoo YL, Delabie J and De Wolf-Peeters C: How reliable is histologic examination of bone marrow trephine biopsy specimens for the staging of non-Hodgkin lymphoma? A study of hairy cell leukemia and mantle cell lymphoma involvement of the bone marrow trephine specimen by histologic, immunohistochemical, and polymerase chain reaction techniques. Am J Clin Pathol 111: 179-184, 1999.

9. Kremer M, Cabras AD, Fend F, et al: PCR analysis of IgH-gene rearrangements in small lymphoid infiltrates microdissected from sections of paraffin-embedded bone marrow biopsy specimens. Hum Pathol 31: 847-853, 2000.

10. Engels K, Oeschger S, Hansmann ML, Hillebrand M and Kriener S: Bone marrow trephines containing lymphoid aggregates from patients with rheumatoid and other autoimmune disorders frequently show clonal B-cell infiltrates. Hum Pathol (In press).

11. Fend F, Gschwendtner A, Gredler E, Thaler J and Dietze O: Detection of monoclonal B-cell populations in decalcified, plastic-embedded bone marrow biopsies with the polymerase chain reaction. Am J Clin Pathol 102: 850-855, 1994.

12. Weirich G, Funk A, Hoepner I, et al: PCR-based assays for the detection of monoclonality in non-Hodgkin's lymphoma: application to formalin-fixed, paraffin-embedded tissue and decalcified bone marrow samples. J Mol Med 73: 235-241, 1995.

13. Wolf J, Kapp U, Bohlen H, et al: Peripheral blood mononuclear cells of a patient with advanced Hodgkin's lymphoma give rise to permanently growing Hodgkin-Reed Sternberg cells. Blood 87: 3418-3428, 1996.

14. Koehler CI, Mues MB, Dienes HP, Kriegsmann J, Schirmacher P and Odenthal M: Helicobacter pylori genotyping in gastric adenocarcinoma and MALT lymphoma by multiplex PCR analyses of paraffin wax embedded tissues. Mol Pathol 56: 36-42, 2003.

15. Brisco MJ, Tan LW, Orsborn AM and Morley AA: Development of a highly sensitive assay, based on the polymerase chain reaction, for rare B-lymphocyte clones in a polyclonal population. Br J Haematol 75: 163-167, 1990. 
16. Segal GH, Jorgensen T, Scott M and Braylan RC: Optimal primer selection for clonality assessment by polymerase chain reaction analysis: II. Follicular lymphomas. Hum Pathol 25: 1276-1282, 1994.

17. Segal GH, Jorgensen T, Masih AS and Braylan RC: Optimal primer selection for clonality assessment by polymerase chain reaction analysis: I. Low grade B-cell lymphoproliferative disorders of nonfollicular center cell type. Hum Pathol 25: $1269-1275,1994$

18. Coad JE, Olson DJ, Christensen DR, et al: Correlation of PCRdetected clonal gene rearrangements with bone marrow morphology in patients with B-lineage lymphomas. Am J Surg Pathol 21: 1047-1056, 1997.

19. Coad JE, Olson DJ, Lander TA and McGlennen RC: Molecular assessment of clonality in lymphoproliferative disorders: I. Immunoglobulin gene rearrangements. Mol Diagn 1: 335-355, 1996.

20. Lehmann U, Bock O, Langer F and Kreipe H: Demonstration of light chain restricted clonal B-lymphoid infiltrates in archival bone marrow trephines by quantitative real-time polymerase chain reaction. Am J Pathol 159: 2023-2029, 2001.

21. Crescenzi M, Seto M, Herzig GP, Weiss PD, Griffith RC and Korsmeyer SJ: Thermostable DNA polymerase chain amplification of $\mathrm{t}(14 ; 18)$ chromosome breakpoints and detection of minimal residual disease. Proc Natl Acad Sci USA 85: 4869-4873, 1988.
22. Martens AC, Schultz FW and Hagenbeek A: Nonhomogeneous distribution of leukemia in the bone marrow during minimal residual disease. Blood 70: 1073-1078, 1987.

23. Schwonzen M, Pohl C, Steinmetz T, et al: Immunophenotyping of low-grade B-cell lymphoma in blood and bone marrow: poor correlation between immunophenotype and cytological/ histological classification. Br J Haematol 83: 232-239, 1993.

24. Crotty PL, Smith BR and Tallini G: Morphologic, immunophenotypic, and molecular evaluation of bone marrow involvement in non-Hodgkin's lymphoma. Diagn Mol Pathol 7: 90-95, 1998.

25. Kang YH, Park CJ, Seo EJ, et al: Polymerase chain reactionbased diagnosis of bone marrow involvement in 170 cases of non-Hodgkin lymphoma. Cancer 94: 3073-3082, 2002.

26. Lust JA: Molecular genetics and lymphoproliferative disorders. J Clin Lab Anal 10: 359-367, 1996.

27. Evans PA, Pott C, Groenen PJ, et al: Significantly improved PCR-based clonality testing in B-cell malignancies by use of multiple immunoglobulin gene targets. Report of the BIOMED-2 Concerted Action BHM4-CT98-3936. Leukemia 21: 207-214, 2007. 